\title{
The Efficacy of an Oral Elemental Diet in Patients Undergoing Hematopoietic Stem Cell Transplantation
}

\author{
Takanobu Morishita ${ }^{1}$, Natsuko Tsushita ${ }^{2}$, Kanae Imai $^{2}$, Toshiyasu Sakai ${ }^{2}$, Kotaro Miyao ${ }^{1}$, \\ Reona Sakemura ${ }^{1}$, Tomonori Kato ${ }^{2}$, Keiko Niimi ${ }^{2}$, Yoshitaka Ono ${ }^{2}$ and Masashi Sawa ${ }^{2}$
}

\begin{abstract}
Objective Conditioning regimens for hematopoietic stem cell transplantation (HSCT) are well known to cause severe gastrointestinal toxicities that often disturb the oral intake of the patients followed by poor nutrition and life-threatening infection. An oral elemental diet (ED) is an easily consumed and assimilated form of liquid nutrients mainly composed of amino acids. It alleviates the digestive loading from the intestine and is mainly used for enteral nutritional support in patients with Crohn's disease. We herein report, for the first time, the efficacy of ED for patients undergoing HSCT.

Methods We evaluated the efficacy of ED in a prospective cohort study. The primary endpoint for this study was the hospitalization period. The secondary endpoint was the occurrence of oral mucositis, nausea, diarrhea and fever.

Patients A total of 73 patients were consecutively enrolled between March 2011 and March 2013. Twentythree patients underwent autologous HSCT and 50 patients underwent allogeneic HSCT. The first 21 patients did not receive ED (non-ED group; NEG) while in the successive 52 patients (ED group; EG), oral ED was started before conditioning and was continued until 28 days after transplantation.

Results The patient characteristics were similar between the two groups. The mean duration of ED administration for EG was 28.7 days (range, 3-37 days), and the mean total-dose of ED administration was $1904 \mathrm{~g}$ (range, 240-2,960 g). The median hospitalization period was significantly shorter in EG compared to NEG, (34 days vs. 50 days; $\mathrm{p}=0.007$ ). Grade $3-4$ oral mucositis occurred less in EG than NEG (25\% vs. $48 \%$; $\mathrm{p}=$ $0.06)$.

Conclusion Oral ED may promote an early mucosal recovery and thereby shorten the duration of hospitalization.
\end{abstract}

Key words: elemental diet, hematopoietic stem cell transplantation, hospitalization, mucositis, GVHD

(Intern Med 55: 3561-3569, 2016)

(DOI: 10.2169/internalmedicine.55.7310)

\section{Introduction}

An elemental diet (ED) is oral liquid formulations of amino acids, providing elemental nitrogen and easily digestible carbohydrates, minerals and vitamins. The amount of fat is minimal and the formulations contain no fiber (Supplementary material 1). ED can be easily administered even in patients with mucosal damage and it has so far been used primarily for patients with inflammatory bowel diseases
(IBD) such as Crohn's Disease and ulcerative colitis. ED for Crohn's disease has shown efficacy equivalent to that of corticosteroids and it may also reduce pathogenic bacteria and maintain species diversity (1).

High dose alkylating agents such as cyclophosphamide (CY), melphalan (Mel), busulfan (BU), and total body irradiation (TBI) which are commonly used in conditioning regimens for hematopoietic stem cell transplantation (HSCT), and methotrexate (MTX) are well-known causes of severe gastrointestinal toxicities including mucositis associ-

${ }^{1}$ Department of Hematology and Oncology, Nagoya University Graduate School of Medicine, Japan and ${ }^{2}$ Department of Hematology and Oncology, Anjo Kosei Hospital, Japan

Received for publication February 22, 2016; Accepted for publication May 5, 2016

Correspondence to Dr. Takanobu Morishita, morishita-ygc@umin.ac.jp 
Table 1. Patient Characteristics.

\begin{tabular}{|c|c|c|c|}
\hline & EG & NEG & $\mathrm{p}$ value \\
\hline Patient, no. (\%) & $52(71)$ & $21(29)$ & 0.71 \\
\hline Median age, y (range) & $47(17-67)$ & $48(26-72)$ & 0.89 \\
\hline$\overline{\text { Sex, no. (\%) }}$ & & & 0.1 \\
\hline Male & $26(50)$ & $15(71)$ & \\
\hline Female & $26(40)$ & $6(29)$ & \\
\hline$\overline{\text { Disease, no. (\%) }}$ & & & 0.11 \\
\hline AML & $14(27)$ & $9(42)$ & \\
\hline ALL & $11(21)$ & $2(10)$ & \\
\hline ML & $16(31)$ & $8(38)$ & \\
\hline MM & $7(13)$ & 0 & \\
\hline ATL & $1(2)$ & $1(5)$ & \\
\hline CML & 0 & $1(5)$ & \\
\hline $\mathrm{AA}$ & $3(6)$ & 0 & \\
\hline$\overline{\text { Disease status at HSCT, no. (\%) }}$ & & & 0.33 \\
\hline $\mathrm{CR}$ & $35(67)$ & $17(81)$ & \\
\hline $\mathrm{PR}$ & $7(14)$ & 0 & \\
\hline $\mathrm{SD} / \mathrm{PD}$ & $10(19)$ & $4(19)$ & \\
\hline Type of HSCT, no. (\%) & & & 0.73 \\
\hline Autologous-HSCT & $17(33)$ & $6(28)$ & \\
\hline Allogeneic-HSCT & $35(67)$ & $15(72)$ & \\
\hline Time to engraftment, median days (range) & $14(9-29)$ & $15(9-29)$ & 0.56 \\
\hline
\end{tabular}

EG: elemental diet group, NEG: non-elemental diet group, AML acute myeloid leukemia, ALL acute lymphoblastic leukemia, ML: malignant lymphoma, MM: multiple myeloma, ATL: adult T-cell leukemia, CML: chronic myeloid leukemia, AA: aplastic anemia, CR: complete remission, PR: partial remission, SD/PD: stable disease/progression disease, HSCT: hematopoietic stem cell transplantation

ated with oral pain (2). Mucositis and pain reduce the patients' oral intake and lead to inadequate nutrition and lifethreatening infections. Inadequate nutrition requiring parenteral nutrition (PN) prolongs hospitalization and lifethreatening infections may result from bacterial translocation through damaged mucosal membranes. Poor oral intake early after HSCT may also be associated with an increased risk of developing severe acute graft vs. host disease (GVHD) $(3,4)$. Severe weight loss of more than $10 \%$ of normal body weight caused by nutritional problems has previously been reported to be a factor for an increased risk of non-relapse mortality and avoiding malnutrition is therefore mandatory $(5,6)$. Indeed, nutrition support by enteral tube feeding after allogeneic HSCT is effective for achieving better outcomes, a prolonged overall survival, good neutrophil/ platelet engraftment, and for avoiding acute GVHD development $(7,8)$.

Proujansky et al. reported that the intestinal mucosa in bone marrow transplant recipients and IBD patients shared pathogenic features, such as major histocompatibility complex expression on intestinal epithelial cells and the local production of cytokines (9). Furthermore, ED is known to have a beneficial effect on the stability of intestinal microbiota. These facts have all been reported to positively contribute to the clinical outcome in allogeneic $\operatorname{HSCT}(1,10)$. To the best of our knowledge, there are no previous reports on the efficacy of oral ED in patients with HSCT. In this study, the patients were prospectively assigned to ED group (EG) and non-ED group (NEG), based on the time period when HSCT was performed. We examined ED by analyzing the differences in hospitalization duration and the short-term transplant outcome between the two groups.

\section{Materials and Methods}

\section{Patients}

We enrolled 73 consecutive patients who underwent HSCT between March 2011 and March 2013 at Anjo Kosei Hospital, Aichi, Japan. We followed up the patients' status until December 2014. All 52 HSCT patients who were enrolled from November 2011 onward received ED as standard care.

\section{Study design}

This study was designed as a prospective cohort study. Among a total of 73 patients enrolled, the first 21 patients did not receive ED and were assigned to NEG. Starting from November 2011, 52 consecutive patients who received ED were assigned to EG. We used Elental (Ajinomoto, Tokyo, Japan) as the ED in our study. ED was administered from day -8 to day -4 , before conditioning regimens, and then it was continued until day +28 after HSCT. For patients who were discharged from the hospital before day +28 , ED 


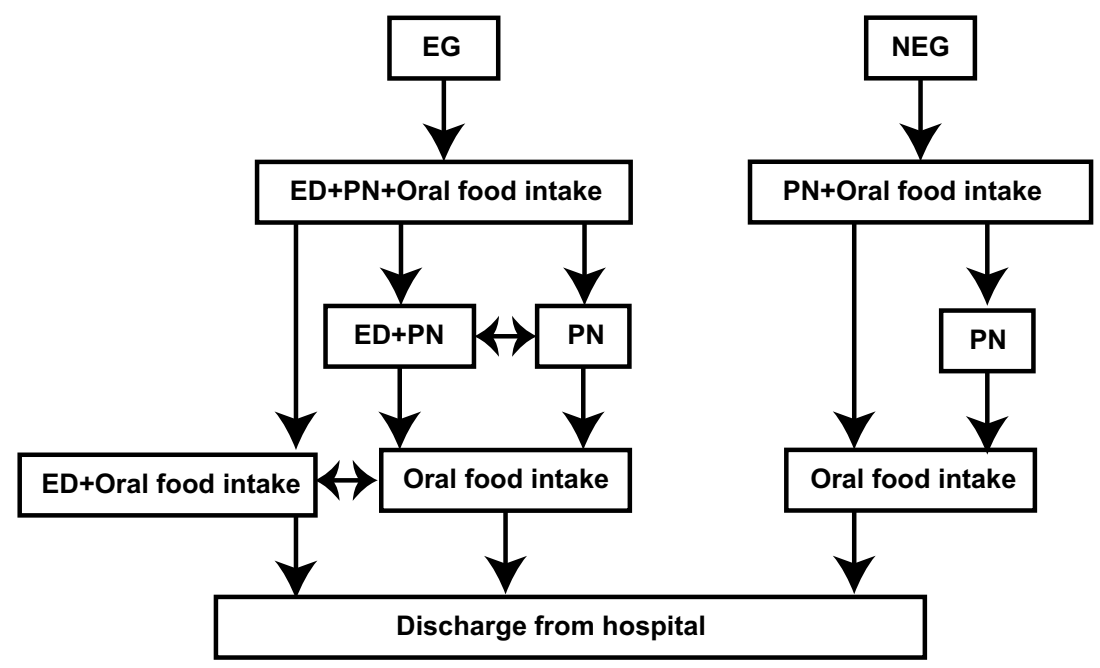

Figure 1. Study design. The study flow chart is shown. The elemental diet (ED) was started from day-8 to day- 4 before transplantation, and it was continued until day+28 after transplantation unless a patient was unable to take ED. Parenteral nutrition (PN) was added to maintain a total calorie intake of $126 \mathrm{~kJ} / \mathrm{kg} / \mathrm{day}$, when food intake and ED administration became insufficient in both the elemental diet group (EG) and non-elemental diet group (NEG). Discharge from hospital was considered as soon as PN support became unnecessary. EG: elemental diet group, NEG: non-elemental diet group, PN: parenteral nutrition, ED: elemental diet

administration was continued on an outpatient basis. The targeted daily dose of ED was $80 \mathrm{~g} /$ body/day $(1,255 \mathrm{~kJ}=300$ $\mathrm{kcal})$. Total-dose is from $2,560 \mathrm{~g}$ to $2,960 \mathrm{~g}$, which varies with the length of the pre-conditioning regimens. The dose presumed suitable for both ingestible adherence and supplemental support (1). ED administration was ceased when the patients were unable to take ED orally. We added PN and/or food intake to adjust the total daily caloric intake to $126 \mathrm{~kJ} /$ $\mathrm{kg} /$ day (30 kcal/kg/day) in both EG and NEG patients, according to the guidelines for the use of parenteral and enteral nutrition (11). For allogeneic HSCT patients, PN was started on day -1 , when almost all patients were unable to consume food. For autologous HSCT patients, PN was started when the patients were unable to reach an oral caloric intake of $126 \mathrm{~kJ} / \mathrm{kg} /$ day. Additional PN was continued until the patients' oral caloric intake reached $126 \mathrm{~kJ} / \mathrm{kg} / \mathrm{day}$ for both allogeneic and autologous patients. The patients were discharged from hospital when the patients required no PN support or no in-hospital care for transplant-related complications (Fig. 1). This study was approved by the Anjo Kosei Hospital review board. Informed consent was obtained from each patient in written form before enrolling in the study.

\section{Transplantation procedure}

Patients undergoing autologous HSCT received LEED (Mel, etoposide, CY, dexamethasone) for malignant lymphoma (12) or high-dose Mel for multiple myeloma as a conditioning regimen (13). Granulocyte-colony stimulating factor (G-CSF) injection was started from the time when the patients' neutrophil counts were under $500 / \mu \mathrm{L}$ and it was continued until the counts reached a level of over $500 / \mu \mathrm{L}$.
For allogeneic HSCT patients, myeloablative conditioning (MAC) or reduced intensity conditioning (RIC) was employed according to age and comorbidity. The MAC regimen consisted of CY + TBI; (14) BU + CY; (15) cytosine arabinoside (CA)+ CY+ TBI (16-18). RIC consisted of fludarabine $(\mathrm{Flu})+\mathrm{Mel} ;(19,20) \mathrm{Flu}+\mathrm{CY}+\mathrm{TBI} ;(21) \mathrm{Flu}+$ BU; (22) Flu + BU + TBI; (23) Flu+ Mel+ TBI (2 Gy); (24) Flu+ CY+ antithymocyte globulin (25). G-CSF injection was started from day 7 and it was continued until the patients' neutrophil counts reached a level of over 500/ $\mu \mathrm{L}$.

\section{GVHD prophylaxis}

Prophylaxis consisted of a combination of tacrolimus (TAC) or cyclosporine (CsA) and a short course of MTX. MTX was administered on day $+1,+3,+6$ at a dose of 15 , 10 , and $10 \mathrm{mg} / \mathrm{m}^{2}$, respectively for patients receiving allogeneic HSCT from a matched unrelated donor (MUD) and for patients receiving cord blood transplantation (CBT), and at a dose of 10,7 , and $7 \mathrm{mg} / \mathrm{m}^{2}$, respectively for patients receiving allogeneic HSCT from a matched related donor (MRD) (26). CsA was administered to patients receiving allogeneic HSCT from an MRD; TAC was administered to patients receiving allogeneic HSCT from an MUD and CBT. Leucovorin was added only in cases of CBT to reduce the mucosal damage induced by MTX.

\section{Histocompatibility leukocyte antigen (HLA) typing}

We performed high resolution typing for HLA-A, -B, -C, and -DR in allogeneic HSCT patients and for HLA-A, -B, and -DR in the MRD and MUD for patients receiving CBT. HLA mismatching was acceptable with at least $7 / 8$ matched 
for allogeneic HSCT patients, and at least 4/6 matched for CBT patients.

\section{Study end-points}

The primary endpoint for this study was the hospitalization period. The duration of hospitalization was defined as the days from transplant to the date of discharge. The secondary endpoint was the occurrence of oral mucositis, nausea, diarrhea and fever. In addition, the cumulative incidence of discharge from hospital at day 50, acute GVHD, and the day 100 non-relapse mortality (NRM) was also analyzed. We evaluated the occurrence and the duration of oral mucositis, nausea, diarrhea, and fever, Regimen-related toxicities were graded according to the criteria proposed by the National Cancer Institute-Common Terminology for Adverse Events v4.0 (CTCAE v4.0). Acute GVHD was diagnosed both clinically and pathologically, and its severity was graded by consensus criteria (27). To minimize any patientto-patient variation, the patients were thoroughly informed of the condition of discharge before enrollment, and the physician's decision regarding the discharge date was monitored during the study period.

\section{Statistical Analysis}

We used Pearson's chi-square test or Fisher's exact test for the nominal scales. Student's $t$-test was used to analyze the parametric continuous scales, and the Mann-Whitney U test was used to compare the non-parametric continuous scales. The cumulative incidence of discharge from hospital was determined by Gray's analysis with death before discharge as a competing risk. The cumulative incidence of acute GVHD and NRM was calculated with relapse and death without GVHD, and relapse as a competing risk, respectively. The analyses were performed on an intention-totreat basis and a $\mathrm{p}$ value $<0.05$ was considered to be significant. All analyses were performed using the SPSS software program ver. 22 (SPSS Inc., Chicago, IL, USA).

\section{Results}

\section{Patient characteristics}

Twenty-three patients underwent autologous HSCT, and 50 patients underwent allogeneic HSCT. The median age at HSCT was 47 years (range, 17-72 years), with 41 males and 32 females included in the analysis. The patients had acute myeloid leukemia $(n=23)$, acute lymphoblastic leukemia $(n=$ $13)$, malignant lymphoma $(n=24)$, multiple myeloma $(n=7)$, adult T-cell leukemia/lymphoma $(\mathrm{n}=2)$, chronic myeloid leukemia $(n=1)$, and aplastic anemia $(n=3)$. Fifty-two patients were in complete remission, 7 patients were in partial remission, and 14 patients had stable disease/disease progression. All of the patients had an Eastern Cooperative Oncology Group performance status of 0-2, and were eligible for autologous HSCT and allogeneic HSCT. The patients were deemed ineligible if they had an Eastern Cooperative Oncol- ogy Group performance status of 3-4, and uncontrolled bacterial infection, fungal infection, cytomegalovirus, hepatitis, or HIV infection.

The baseline demographics of the study participants were comparative between EG and NEG (Table 1). There were no patients with aplastic anemia (AA) and multiple myeloma $(\mathrm{MM})$ in EG. The pre-HSCT disease statuses between EG and NEG were as follows: complete remission (CR) $(67 \%$ vs. $81 \%$ ), partial response (PR) (14\% vs. $0 \%)$ and stable disease/progressive disease (SD/PD) (19\% vs. 19\%). The median days to engraftment were 14 days (range, 9-29 days) among EG and 15 days (range, 9-29 days) among NEG. The conditioning regimen, TBI, donor source, HLA matching, and GVHD prophylaxis were comparative for the two groups (Table 2) and further information about condition regimens of allogeneic HSCT are shown in Supplementary material 2. In EG, $26 \%$ of patients received 12 Gy-TBI regimens in comparison to $47 \%$ in NEG $(p=0.17)$. The differences in the donor source among EG and NEG were as follows: unrelated bone marrow (26\% vs. $47 \%$ ), related peripheral blood stem cell (40\% vs.13\%) and unrelated cord blood (34\% vs. $40 \%)$. The differences were not statistically different.

\section{ED administration}

The mean duration of ED administration for EG was 28.7 days (range, 3-37 days). The mean total-dose and the mean dose-per-day of ED administration was 1,904 g (range, 240$2,960 \mathrm{~g}$ ) and $65 \mathrm{~g} /$ day (range, 14.1-80 g/day), respectively. The mean duration of additional oral food intake was 22.7 days (range, 2-37 days) for EG and 21 days (range, 3-35 days) for NEG ( $\mathrm{p}=0.9)$.

\section{Hospitalization duration}

The median duration of hospitalization after transplantion was 40 days (range, 14-116 days). When stratified by ED administration, the median duration was significantly shorter in EG compared with NEG (34 days vs. 50 days; $\mathrm{p}=0.007$ ) (Fig. 2A). The cumulative incidence of discharge from hospital at day 50 was $64 \%$ in EG and $43 \%$ in NEG. The difference was not significant ( $\mathrm{p}=0.07$ ) (Fig. 3A).

When the analysis was performed for patients who received allogeneic HSCT, the median hospitalization duration was also significantly shorter in EG compared to NEG (41.5 days vs. 61 days; $\mathrm{p}=0.0002$ ) (Fig. $2 \mathrm{~B}$ ). The cumulative incidence of discharge from hospital at day 50 was $51 \%$ in EG and $33 \%$ in NEG (p=0.11) (Fig. 3B).

\section{Transplant outcome}

Grade 3-4 oral mucositis occurred in 13 (25\%) EG patients and 10 (48\%) NEG patients $(\mathrm{p}=0.06)$. The mean duration of mucositis in EG and NEG patients was 2.8 days and 5.6 days $(\mathrm{p}=0.07)$, respectively. Grade $2-4$ fever occurred in $43(83 \%)$ EG patients and $14(67 \%)$ NEG patients ( $\mathrm{p}=0.13)$, and the mean durations of the fever were 3.9 days and 5.0 days, respectively $(\mathrm{p}=0.95)$. Documented infections were ob- 
Table 2. Allogeneic Hematopoietic Stem Cell Transplantation Characteristics.

\begin{tabular}{|c|c|c|c|}
\hline & EG & NEG & $\mathrm{p}$ value \\
\hline Conditioning regimen, no. (\%) & & & 0.36 \\
\hline MAC & $15(43)$ & $9(60)$ & \\
\hline RIC & $20(57)$ & $6(40)$ & \\
\hline TBI conditions, no. (\%) & & & 0.12 \\
\hline $12 \mathrm{~Gy}$ & $10(29)$ & $7(47)$ & \\
\hline 2 Gy & $8(23)$ & 0 & \\
\hline non-TBI & $17(49)$ & $8(53)$ & \\
\hline Donor source, no. (\%) & & & 0.17 \\
\hline Unrelated bone marrow & $9(26)$ & $7(47)$ & \\
\hline Related PBSC & $14(40)$ & $2(13)$ & \\
\hline Unrelated cord blood & $12(34)$ & $6(40)$ & \\
\hline HLA matching, no. (\%) & & & 0.4 \\
\hline Full match & $23(66)$ & $9(60)$ & \\
\hline $5 / 6$ cord blood mismatch & $1(3)$ & $2(13)$ & \\
\hline $4 / 6$ cord blood mismatch & $11(31)$ & $4(27)$ & \\
\hline GVHD prophylaxis, no. (\%) & & & 0.16 \\
\hline sMTX+TAC & $9(26)$ & $8(53)$ & \\
\hline $\mathrm{sMTX}+\mathrm{CsA}$ & $14(40)$ & $2(13)$ & \\
\hline $\mathrm{sMTX}+\mathrm{TAC}+\mathrm{LV}$ & $12(34)$ & $5(34)$ & \\
\hline
\end{tabular}

HSCT: hematopoietic stem cell transplantation, EG: elemental diet group, NEG: non-elemental diet group,

MAC: myeloablative conditioning, RIC reduced intensity conditioning, TBI: total body irradiation, Gy: grays,

HLA: histocompatibility leukocyte antigen, PBSC: peripheral blood stem cell, GVHD: graft vs. host disease,

sMTX: short course methotrexate, TAC: tacrolimus, CsA: cyclosporine, LV: leucovorin

A

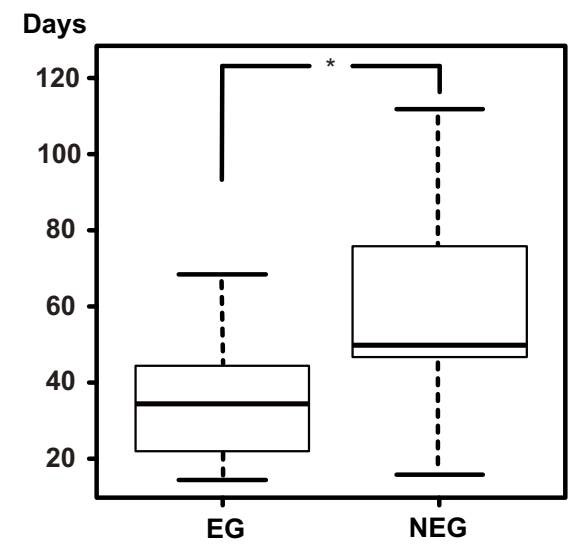

B

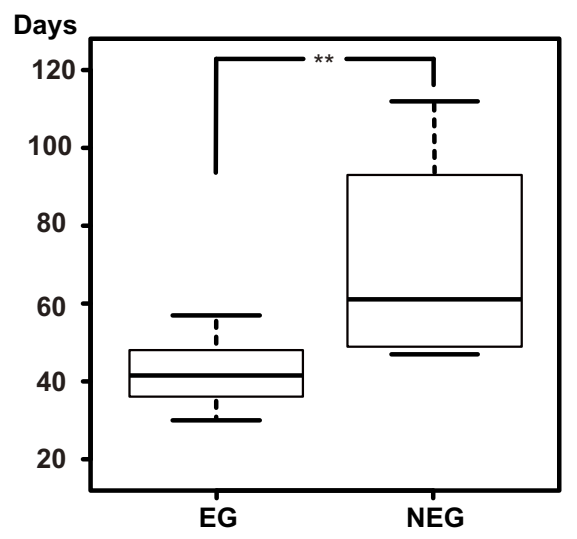

Figure 2. Hospitalization duration. The durations of hospitalization for the elemental diet group (EG) and non-elemental diet group (NEG) are shown in a box plot. No differences between EG and NEG are demonstrated in (A): all patients and in (B): patients with allogeneic HSCT. A bold horizontal line in a box plot indicates the median days of hospitalization. The duration of hospitalization periods were significantly short in EG. EG: elemental diet group, NEG: non-elemental diet group. : $\mathbf{p}<0.05 ;^{* *}: \mathbf{p}<0.001$.

served in $4(7.7 \%)$ EG patients and 1 (4.8\%) NEG patients $(\mathrm{p}=0.67)$. The occurrence of sever organ failure between $E G$ and NEG were $7(13.4 \%)$ vs. $5(23.8 \%)$ as detailed $(\mathrm{p}=0.29)$; acute respiratory distress syndrome (3 (5.8\%) vs. 3 $(14.3 \%))$, sinusoidal obstructive syndrome (2 $(3.8 \%)$ vs. 0 $(0 \%))$, hemorrhagic cystitis $(0(0 \%)$ vs. $2(9.5 \%))$ and engraftment failure $(2(3.8 \%)$ vs. $0(0 \%))$. There were no significant differences in the use of opioid agents, grade 3-4 diarrhea, or grade 3-4 nausea, for both frequency and duration
(Table 3). The cumulative incidence of grade II-IV acute GVHD at day 100 was $15 \%$ for EG patients and $30 \%$ for NEG patients, but the difference was not statistically significant $(\mathrm{p}=0.26)$. The cumulative incidence of day 100 nonrelapse mortality was $9.6 \%$ for EG patients and $14.3 \%$ for NEG patients $(\mathrm{p}=0.9)$. 

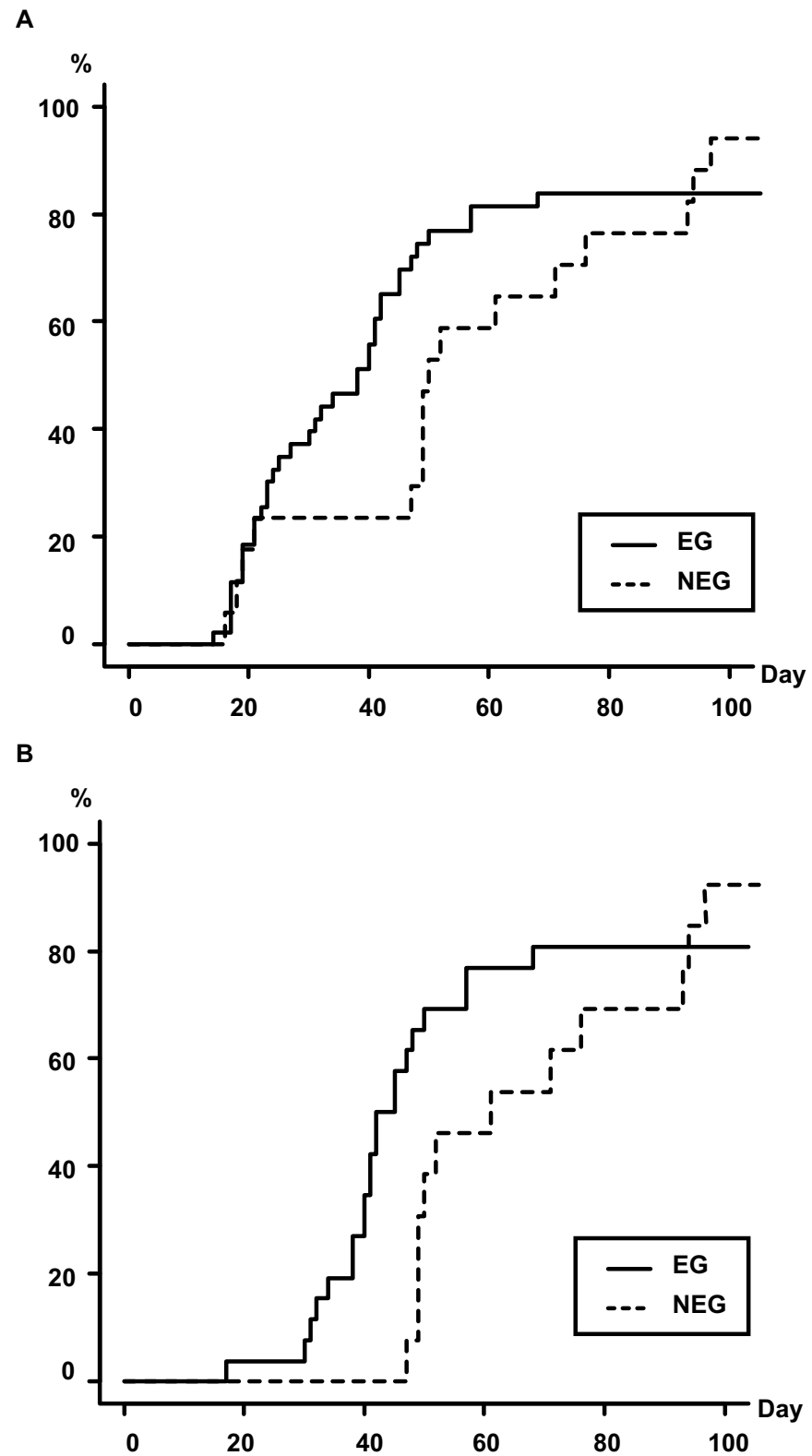

Figure 3. Cumulative incidence of hospital discharge. The cumulative incidence curves of the days from transplantation to hospital discharge are shown. A solid line indicates the elemental diet group (EG) and a dashed line indicates the non-elemental diet group (NEG). The incidence of each group is shown in (A): all patients and in (B): patients with allogeneic HSCT. The difference was not significant between EG and NEG, but EG did tend to have a shorter hospitalization period. EG: elemental diet group, NEG: non-elemental diet group

\section{Discussion}

Our study demonstrated a significant reduction in the hospitalization period in patients who received oral ED compared to those who did not. Many factors are involved regarding the hospitalization period for HSCT patients. Early transplant-related complications including regimen-related toxicities and acute GVHD were major obstacles that prevent early hospital discharge (28-30). Although the incidence of those complications were not significantly different between EG patients and NEG patients, a trend was ob- served, namely that the patients who received ED had less grade 3-4 oral mucositis. On the other hand, the incidence of patients with diarrhea was higher in EG. It was considered to be an adverse event of ED intake, not due to any mucosal damage, but due to its osmotic balance. The mean duration time of diarrhea was not long enough to lead to ED discontinuation. The ED intake might be associated with early intestinal mucosa recovery, which helped to accelerate oral food intake after transplantation and thus allow for an earlier hospital discharge. ED might therefore play an important role in intestinal epithelial barrier support.

The pathogenesis of epithelial barrier dysfunction is a 
Table 3. Transplant outcome.

\begin{tabular}{lcccc}
\hline & & EG & NEG & p value \\
\hline Oral mucositis (grade 3-4) & No. (\%) & $13(25)$ & $10(48)$ & 0.06 \\
& Mean duration (days) & 2.8 & 5.6 & 0.07 \\
\hline Fever (grade 2-4) & No. (\%) & $43(83)$ & $14(67)$ & 0.13 \\
& Mean duration (days) & 3.9 & 5 & 0.95 \\
\hline Diarrhea (grade 3-4) & No. (\%) & $25(50)$ & $6(26)$ & 0.08 \\
& Mean duration (days) & 5 & 2.1 & 0.13 \\
\hline Nausea (grade 3-4) & No. (\%) & $14(26)$ & $8(35)$ & 0.35 \\
& Mean duration (days) & 5.8 & 3.3 & 0.36 \\
\hline Opioid agent use & No. (\%) & $29(58)$ & $11(48)$ & 0.7 \\
& Mean duration (days) & 6.2 & 8.3 & 0.41 \\
\hline
\end{tabular}

lack of intestinal epithelial integrity and tissue viability leading to an increased epithelial permeability, subsequently inducing bacterial translocation. Intestinal microbiota and intestinal immune systems are major responsible factors for epithelial barrier dysfunction. As a supportive nutrient among patients after HSCT, usual diets or oligopeptides or amino acids (major contents of ED) are considered to be important. The mechanisms associated with ED remain unclear, but potential benefits have been observed. First of all, unlike usual diets, ED is a low fat diet and it might be less of a burden for the intestinal mucosa. Secondly, ED might increase and stabilize the intestinal bacterial micro biota as reported in patients with Crohn's disease (1). It would be helpful to investigate the change in the intestinal bacterial flora in HSCT patients. Finally, unlike oligopeptides, amino acids are the final digested form of protein with a loss of antigenicity and thus are less inflammatory for the intestinal mucosa $(31,32)$. Recently, amino acids have been reported to contribute to the regulation of the intestinal immune system in IBD (31-34). One major amino acid, glycine is a nonessential amino acid with many functions. It plays a protective role in renal injuries induced by CsA and liver injuries induced by endotoxin, and it also has an immunosuppressive nature by inhibiting tumor necrosis factor (TNF)$\alpha$ (34). A previous paper reported that the intestinal mucosa in the bone marrow transplant patients and IBD patients had common features regarading the main pathogenic events, such as major histocompatibility complex expression in intestinal epithelial cells and the local production of cytokines (9).

Palifermin, a recombinant human keratinocyte growth factor, has effectively reduced the duration and severity of oral mucositis after intensive chemotherapy and radiation therapy followed by both autologous and allogeneic HSCT for hematological malignancies (35-39). Although, the administration of palifermin has so far failed to show any reduction of acute GVHD, an advantage was observed in days of PN and the length of hospitalization among patients who received TBI $(3,39-42)$. The combined use of ED and palifermin might thus be a potential strategy for providing additional benefits.

The present study is associated with several limitations. This study was performed in a single institution and included both autologous and allogeneic patients. In addition, the small patient number reduced the statistical power and the non-randomized study design hampered the interpretation of the results. AA and MM patients, who are generally expected to demonstrate less gastrointestinal toxicities were only included in EG. The number of patients who received 12 Gy-TBI and the patients transplanted from an unrelated donor source, who are generally expected to have a higher risk of mucosal damage was relatively higher in NEG. On the other hand, the number of patients with a worse disease status were rather high in EG. The incidence of other HSCT-associated complications, such as documented infections or sever organ failure, were not significantly different between two groups, but the incidence of severe organ failure was rather high in NEG. Nevertheless, the prospective nature of this study and the consecutive enrollment with a short period is considered to have allowed us to gain new insight into a novel field in HSCT. Even though the discharge date was determined on a protocol basis, an intentional shortening or extension of hospitalization was not completely eliminated.

In summary, this preliminary study indicated that administering oral ED may shorten the hospitalization duration by reducing the occurrence of intestinal mucositis and it is therefore expected to be a beneficial modality for patients undergoing HSCT. Large prospective randomized placebocontrolled trials are called for in the future to confirm our findings.

The authors state that they have no Conflict of Interest (COI). 


\section{Acknowledgement}

We would like to thank the patients and their families for participating in this study. Without them, this study could not have been carried out.

\section{Ethical Approval}

All procedures performed in studies involving human participants were in accordance with the ethical standards of the institutional and/or national research committee and with the 1964 Helsinki declaration and its later amendments or comparable ethical standards. This study was approved by the Anjo Kosei Hospital review board. Informed consent was obtained from each patient in written form before enrolling in the study. We confirm that all ongoing and related trials for this drug are registered.

\section{References}

1. Shiga H, Kajiura T, Shinozaki J, et al. Changes of faecal microbiota in patients with Crohn's disease treated with an elemental diet and total parenteral nutrition. Dig Liver Dis 44: 736-742, 2012.

2. Cutler C, Li S, Kim HT, et al. Mucositis after allogeneic hematopoietic stem cell transplantation: a cohort study of methotrexateand non-methotrexate-containing graft-versus-host disease prophylaxis regimens. Biol Blood Marrow Transplant 11: 383-388, 2005.

3. Jagasia MH, Abonour R, Long GD, et al. Palifermin for the reduction of acute GVHD: a randomized, double-blind, placebocontrolled trial. Bone Marrow Transplant 47: 1350-1355, 2012.

4. Mattsson J, Westin S, Edlund S, Remberger M. Poor oral nutrition after allogeneic stem cell transplantation correlates significantly with severe graft-versus-host disease. Bone Marrow Transplant 38: 629-633, 2006.

5. Fuji S, Mori T, Khattry N, et al. Severe weight loss in 3 months after allogeneic hematopoietic SCT was associated with an increased risk of subsequent non-relapse mortality. Bone Marrow Transplantation 50: 100-105, 2015.

6. Fuji S, Einsele H, Savani BN, Kapp M. Systematic nutritional support in allogeneic hematopoietic stem cell transplant recipients. Blood Marrow Transplant 21: 1707-1713, 2015.

7. Seguy D, Duhamel A, Rejeb MB, et al. Better outcome of patients undergoing enteral tube feeding after myeloablative conditioning for allogeneic stem cell transplantation. Transplantation 94: 287294, 2012.

8. Seguy D, Berthon C, Micol JB, et al. Enteral feeding and early outcomes of patients undergoing allogeneic stem cell transplantation following myeloablative conditioning. Transplantation 82: 835-839, 2006.

9. Proujansky R. Fixing the intestinal mucosa in the bone marrow transplant patient: lessons from other intestinal immunodeficiencies and inflammatory disorders. Pediatric Transplantation 3 (Suppl 1): 9-13, 1999.

10. Taur Y, Jenq RR, Perales MA, et al. The effects of intestinal tract bacterial diversity on mortality following allogeneic hematopoietic stem cell transplantation. Blood 124: 1174-1182, 2014.

11. Guidelines for the use of parenteral and enteral nutrition in adult and pediatric patients. American Society for Parenteral and Enteral Nutrition. JPEN J Parenter Enteral Nutr 17(4 Suppl): 1SA-52SA, 1993.

12. Han LN, Zhou J, Hirose T, Imai Y, Ishiguro T, Chou T. Feasibility and efficacy of high-dose melphalan, cyclophosphamide, etoposide, and dexamethasone (LEED) chemotherapy with or without rituximab followed by autologous stem cell transplantation for aggressive and relapsed non-Hodgkin's lymphoma. Int J Hematol 84: 174-181, 2006.
13. Harousseau JL, Moreau P. Autologous hematopoietic stem-cell transplantation for multiple myeloma. N Eng J Med 360: 26452654, 2009.

14. Thomas ED Sr. Stem cell transplantation: past, present and future. Stem Cells 12: 539-544, 1994.

15. Geller RB, Saral R, Piantadosi S, et al. Allogeneic bone marrow transplantation after high-dose busulfan and cyclophosphamide in patients with acute nonlymphocytic leukemia. Blood 73: 22092218, 1989.

16. Jillella AP, Doria R, Khan K, et al. Cyclophosphamide, cytosine arabinoside and TBI as a conditioning regimen for allogeneic bone marrow transplantation in patients with leukemia. Bone Marrow Transplant 23: 1095-1100, 1999.

17. Inamoto Y, Nishida T, Suzuki R, et al. Significance of additional high-dose cytarabine in combination with cyclophosphamide plus total body irradiation regimen for allogeneic stem cell transplantation. Bone Marrow Transplant 39: 25-30, 2007.

18. Mori T, Aisa Y, Kato J, et al. Safety and efficacy of total body irradiation, cyclophosphamide, and cytarabine as a conditioning regimen for allogeneic hematopoietic stem cell transplantation in patients with acute lymphoblastic leukemia. Am J Hematol 87: 349-353, 2012.

19. Giralt S, Thall PF, Khouri I, et al. Melphalan and purine analogcontaining preparative regimens: reduced-intensity conditioning for patients with hematologic malignancies undergoing allogeneic progenitor cell transplantation. Blood 97: 631-637, 2001.

20. Inamoto $\mathrm{Y}$, Oba $\mathrm{T}$, Miyamura $\mathrm{K}$, et al. Stable engraftment after a conditioning regimen with fludarabine and melphalan for bone marrow transplantation from an unrelated donor. Int J Hematol 83: 356-362, 2006.

21. Brunstein CG, Barker JN, Weisdorf DJ, et al. Umbilical cord blood transplantation after nonmyeloablative conditioning: impact on transplantation outcomes in 110 adults with hematologic disease. Blood 110: 3064-3070, 2007.

22. Komatsu T, Narimatsu H, Yoshimi A, et al. Successful engraftment of mismatched unrelated cord blood transplantation following reduced intensity preparative regimen using fludarabine and busulfan. Ann Hematol 86: 49-54, 2007.

23. Onishi Y, Mori S, Kusumoto S, et al. Unrelated-donor bone marrow transplantation with a conditioning regimen including fludarabine, busulfan, and $4 \mathrm{~Gy}$ total body irradiation. Int J Hematol 85: 256-263, 2007.

24. Yamamoto H, Kato D, Uchida N, et al. Successful sustained engraftment after reduced-intensity umbilical cord blood transplantation for adult patients with severe aplastic anemia. Blood 117: 3240-3242, 2011.

25. Maury S, Bacigalupo A, Anderlini P, et al. Improved outcome of patients older than 30 years receiving HLA-identical sibling hematopoietic stem cell transplantation for severe acquired aplastic anemia using fludarabine-based conditioning: a comparison with conventional conditioning regimen. Haematologica 94: 1312-1315, 2009.

26. Morishima Y, Morishita $Y$, Tanimoto M, et al. Low incidence of acute graft-versus-host disease by the administration of methotrexate and cyclosporine in Japanese leukemia patients after bone marrow transplantation from human leukocyte antigen compatible siblings; possible role of genetic homogeneity. The Nagoya Bone Marrow Transplantation Group. Blood 74: 2252-2256, 1989.

27. Przepiorka D, Weisdorf D, Martin P, et al. 1994 Consensus Conference on Acute GVHD Grading. Bone Marrow Transplant 15: 825-828, 1995.

28. Legert KG, Remberger M, Ringden O, Heimdahl A, Dahllof G. Reduced intensity conditioning and oral care measures prevent oral mucositis and reduces days of hospitalization in allogeneic stem cell transplantation recipients. Supportive care in cancer: official journal of the Multinational Association of Supportive Care in 
Cancer 22: 2133-2140, 2014

29. Horsley P, Bauer J, Gallagher B. Poor nutritional status prior to peripheral blood stem cell transplantation is associated with increased length of hospital stay. Bone Marrow Transplant 35: 1113$1116,2005$.

30. Mendes ET, Dulley F, Basso M, et al. Healthcare-associated infection in hematopoietic stem cell transplantation patients: risk factors and impact on outcome. Int J Infect Dis 16: e424-e428, 2012.

31. Meister D, Bode J, Shand A, Ghosh S. Anti-inflammatory effects of enteral diet components on Crohn's disease-affected tissues in vitro. Dig Liver Dis 34: 430-438, 2002.

32. Yamamoto $T$, Nakahigashi $M$, Umegae $S$, Kitagawa $T$, Matsumoto $\mathrm{K}$. Impact of elemental diet on mucosal inflammation in patients with active Crohn's disease: cytokine production and endoscopic and histological findings. Inflamm Bowel Dis 11: 580-588, 2005.

33. Andou A, Hisamatsu T, Okamoto S, et al. Dietary histidine ameliorates murine colitis by inhibition of proinflammatory cytokine production from macrophages. Gastroenterology 136: $564-574$ e2, 2009.

34. Tsune I, Ikejima K, Hirose $\mathrm{M}$, et al. Dietary glycine prevents chemical-induced experimental colitis in the rat. Gastroenterology 125: 775-785, 2003.

35. Spielberger R, Stiff P, Bensinger W, et al. Palifermin for oral mucositis after intensive therapy for hematologic cancers. N Engl $\mathrm{J}$ Med 351: 2590-2598, 2004.

36. Abidi MH, Agarwal R, Tageja N, et al. A phase I dose-escalation trial of high-dose melphalan with palifermin for cytoprotection followed by autologous stem cell transplantation for patients with multiple myeloma with normal renal function. Biol Blood Marrow Transplant 19: 56-61, 2013.

37. Elting LS, Shih YC, Stiff PJ, et al. Economic impact of palifermin on the costs of hospitalization for autologous hematopoietic stemcell transplant: analysis of phase 3 trial results. Biol Blood Marrow Transplant 13: 806-813, 2007.

38. Horsley P, Bauer JD, Mazkowiack R, Gardner R, Bashford J. Palifermin improves severe mucositis, swallowing problems, nutrition impact symptoms, and length of stay in patients undergoing hematopoietic stem cell transplantation. Support Care Cancer 15: 105-109, 2007.

39. Nguyen DT, Shayani S, Palmer J, et al. Palifermin for prevention of oral mucositis in allogeneic hematopoietic stem cell transplantation: a single-institution retrospective evaluation. Support Care Cancer 23: 3141-3147, 2015.

40. Panoskaltsis-Mortari A, Taylor PA, Rubin JS, et al. Keratinocyte growth factor facilitates alloengraftment and ameliorates graftversus-host disease in mice by a mechanism independent of repair of conditioning-induced tissue injury. Blood 96: 4350-4356, 2000.

41. Levine JE, Blazar BR, DeFor T, Ferrara JL, Weisdorf DJ. Longterm follow-up of a phase I/II randomized, placebo-controlled trial of palifermin to prevent graft-versus-host disease (GVHD) after related donor allogeneic hematopoietic cell transplantation (HCT). Biology Blood Marrow Transplant 14: 1017-1021, 2008.

42. Goldberg JD, Zheng J, Castro-Malaspina H, et al. Palifermin is efficacious in recipients of TBI-based but not chemotherapy-based allogeneic hematopoietic stem cell transplants. Bone Marrow Transplant 48: 99-104, 2013.

The Internal Medicine is an Open Access article distributed under the Creative Commons Attribution-NonCommercial-NoDerivatives 4.0 International License. To view the details of this license, please visit (https://creativecommons.org/licenses/ by-nc-nd/4.0/).

(C) 2016 The Japanese Society of Internal Medicine http://www.naika.or.jp/imonline/index.html 